\title{
Exposure Method
}

National Cancer Institute

\section{Source}

National Cancer Institute. Exposure Method. NCI Thesaurus. Code C119826.

The technique used to administer the exposure assessment. 